the phenomenon in more depth as they sought to understand its effects in the microscopic world and determine the implications. The authors observed that the contraction generates long-range fluid flows around the organism. When they used beads to visualize the flows, they found that they look like vortices that expand over time. Using well-established equations that describe fluid motion ${ }^{9}$, the authors recapitulated this flow pattern in a computer simulation. This showed that, in a sufficiently liquid medium, the contraction could generate flow that facilitated the dispersal of material around the protist.

But what triggers the contraction? Biologists had thought of this behaviour as a type of 'startle' response, perhaps a reaction to the presence of a predator. However, the exact mechanism had remained elusive. Mathijssen and colleagues propose that the trigger for contraction could be flows in the surrounding fluid generated by the organisms themselves, or by predators.

To test their hypothesis, they constructed an apparatus in which individual S. ambiguum cells were exposed to fluid flows at increasing velocities. Using fluid-dynamics methods ${ }^{10}$, the authors could relate the velocity of the flow that triggers a contraction to the level of tension that such flows induce in biological membranes. They find a remarkable similarity between the level of flow-induced tension that triggers contraction and the tension required to open mechanosensitive ion channels in cell membranes ${ }^{11}$. It has been suggested that protists use mechanosensitive channels to sense liquid flows ${ }^{12}$, and this convergence of theory and experimental work suggests that, once flows reach a certain threshold of magnitude, a contraction is triggered.

At this point, however, the happy union of biology and physics ends. This is because, to satisfy a biologist that such a mechanism is at play, evidence would be required that depletion of these mechanosensitive channels in S. ambiguum cells affects the organisms' ability to react to the liquid flow. Such manipulations are not currently feasible in S. ambiguum, and so, for now, we must regard this mechanism as not definitively proven.

Regardless of the exact molecular details of how cells contract, Mathijssen and colleagues' experiments have clearly established that flows can trigger the contraction. Given their observation that the contraction itself generates flows, the authors then asked what happens when many $S$. ambiguum cells come together in close proximity. Can they trigger each other's contractions by means of the flows they generate? Remarkably, they can. S. ambiguum cells cultured in vitro tend to self-assemble into clusters, and the authors report that, when the cells reach a certain density, the protists exhibit a striking collective phenomenon: one cell spontaneously contracts, triggering contractions in its neighbours and thereby propagating a wave of

contraction through the colony (Fig. 1).

These waves travel at remarkably high speeds, of $0.25 \mathrm{~m} \mathrm{~s}^{-1}$. The authors used further powerful theoretical frameworks ${ }^{13-15}$ to simulate the triggering and propagation of the waves, generating different simulations depending on the orientation, shape and density of the S. ambiguum cells. They compared these theoretical analyses with their experimental results, and established the threshold cellular density at which S. ambiguum colonies are likely to generate fast collective contractions.

But the million-dollar question remains: what is this collective contraction useful for? The authors show, through simulations, that one possible function

"Contraction generates long-range fluid flows around the organism." of waves of contraction is to enable a synchronized discharge of toxins in response to a predator. The flows generated by a large predator itself, or by a predator's attempt to eat an individual S. ambiguum ${ }^{16}$, might generate an initial trigger that induces widespread toxin release by the colony into the surrounding medium. Testing this hypothesis would require targeted genetic manipulations to uncouple the flow-generation and -sensing process from the toxin-release event.

Many protists exhibit rapid contractions, and many cells and organisms, such as bacteria and fishes, generate and sense liquid flows. Investigating the generation and sensing of these flows is likely to be a fruitful topic for future research. lt will be interesting to see how interdisciplinary cooperation between biology and physics continues to shed light on this remarkable signal-propagation process.

Pavel Tomancak is at the Max Planck Institute of Molecular Cell Biology and Genetics,

Dresden 01307, Germany.

e-mail:tomancak@mpi-cbg.de

1. Mathijssen, A. J. T. M., Culver, J., Bhamla, M. S. \& Prakash, M. Nature 571, 560-564 (2019).

2. Buonanno, F., Guella, G., Strim, C. \& Ortenzi, C. Hydrobiologia 684, 97-107 (2012).

3. Haeckel, E. Das protistenreich. Eine populäre uebersicht über das formengebiet der niedersten lebewesen (Günther, 1878); https://doi. org/10.5962/bhl.title.58542

4. Jones, A. R., Jahn, T. L. \& Fonseca, J. R. J. Cell. Physiol. 68, 127-133 (1966).

5. Hawkes, R. B. \& Holberton, D. V. J. Cell. Physiol. 84 225-235 (1974).

6. Lehman, W. J. \& Rebhun, L. I. Protoplasma 72 153-178 (1971).

7. Ishida, H. \& Shigenaka, Y. Cytoskeleton 9, 278-282 (1988).

8. Yagiu, R. \& Shigenaka, Y. J. Protozool. 10, 364-369 (1963).

9. Winkler, R. G. Eur. Phys. J. Spec. Top. 225, 2079-2097 (2016)

10.Cortez, R., Fauci, L. \& Medovikov, A. Phys. Fluids 17, 031504 (2005)

11.Phillips, R., Kondev, J., Theriot, J. \& Garcia, H. G. Physical Biology of the Cell (Garland, 2012).

12. Ohmura, T. et al. Proc. Natl Acad. Sci. USA 115, 3231-3236 (2018).

13.Xia, W. \& Thorpe, M. F. Phys. Rev. A 38, 2650-2656 (1988).

14. Takeuchi, K. A., Kuroda, M., Chaté, H. \& Sano, M. Phys. Rev. Lett. 99, 234503 (2007).

15. Balanis, C. A. Proc. IEEE 80, 7-23 (1992).

16. Buonanno, F. Biologia 66, 648-653 (2011).

\title{
OCEAN SCIENCE
}

\section{Marine heatwaves in a changing climate}

\begin{abstract}
Heatwaves in the ocean can rapidly disrupt marine ecosystems and the economies that depend on them. A global analysis of these events casts light on their causes and sets the stage for revealing how they might change in the future.
\end{abstract}

\section{MICHAEL G. JACOX}

$\mathrm{H}$ eatwaves that occur over land are well known for having adverse impacts on human health, infrastructure and agriculture. Less attention has been paid to analogous episodes in the ocean, dubbed marine heatwaves (MHWs), but interest in these transient events is growing as their potentially dramatic ecological and economic impacts ${ }^{1}$ have become clear. This enhanced awareness of the importance of MHWs has fostered a desire to understand their causes and whether they can be predicted. Writing in Nature
Communications, Holbrook et al. ${ }^{2}$ present the first comprehensive analysis of MHWs across the globe. They identify specific drivers of these events, as well as associations between MHWs and known climate oscillations.

On a local scale, MHWs can be induced by anomalous ocean heating at the ocean surface (caused by changes in air temperature, winds or cloud cover, for example), or as a result of horizontal or vertical currents and mixing in the surrounding ocean. These local MHWdriving processes are often tied to large-scale climate oscillations, and Holbrook et al. have mapped out such relationships globally for 
MHWs that occurred between 1982 and 2016. For example, the authors show that some of the most intense MHWs have occurred in the eastern tropical Pacific Ocean during El Niño events - the warm phase of the El NiñoSouthern Oscillation (ENSO), which involves episodic warming and cooling of the tropical Pacific's surface waters.

Although the centre of action for ENSO is in the eastern tropical Pacific, Holbrook and colleagues' analyses show that its geographical influence is much broader: not only is ENSO the strongest driver of MHWs in much of the Pacific, but also in large parts of the Indian, Atlantic and Southern oceans (Fig. 1). Such a broad influence is made possible by teleconnections - atmospheric and oceanic pathways through which climate signals can be communicated over thousands of kilometres.

One key motivation for understanding the drivers of MHWs is to determine how they can be used to predict these events. Such efforts can also draw on past research into predicting ocean temperatures more generally. ENSO has previously been identified as a prominent source of predictability, enabling forecasts of atmospheric and oceanic variables such as precipitation and temperature ${ }^{3,4}$ up to a year in advance. Holbrook et al. suggest that MHWs in many regions could also potentially be predicted on the basis of their connection to ENSO and other climate oscillations. By contrast, intense MHWs associated with ocean currents off the east coasts of Japan, North America and Australia are mainly related to the chaotic nature of the flow in those regions and are less likely to be predictable.

Among the MHW drivers listed by Holbrook et al. is long-term climate change, and this raises a key caveat that also applies to other papers published on this topic. To explain, it should first be noted that ocean temperatures during MHWs are much higher than 'normal' temperatures, which themselves will shift as the ocean warms with climate change. In other words, the baseline ocean temperature will shift, and future MHWs should therefore be defined relative to the shifted baseline.

However, several studies (see refs 5 and 6, for example) that describe how MHWs are affected by humans' influence on the climate have defined these events relative to ocean temperatures during a fixed period of time, rather than relative to the shifting baseline. These studies concluded that climate change greatly increases the intensity, frequency and persistence of MHWs. However, their findings mostly reflect a warming trend in the ocean rather than a change in MHWs themselves ${ }^{6,7}$; to put it another way, the mean ocean temperature rises, but the variability around the mean temperature stays relatively steady. Fundamentally, defining MHWs without adjusting for a warming trend conflates regional climate variability with global climate change. Ironically, the same type of argument

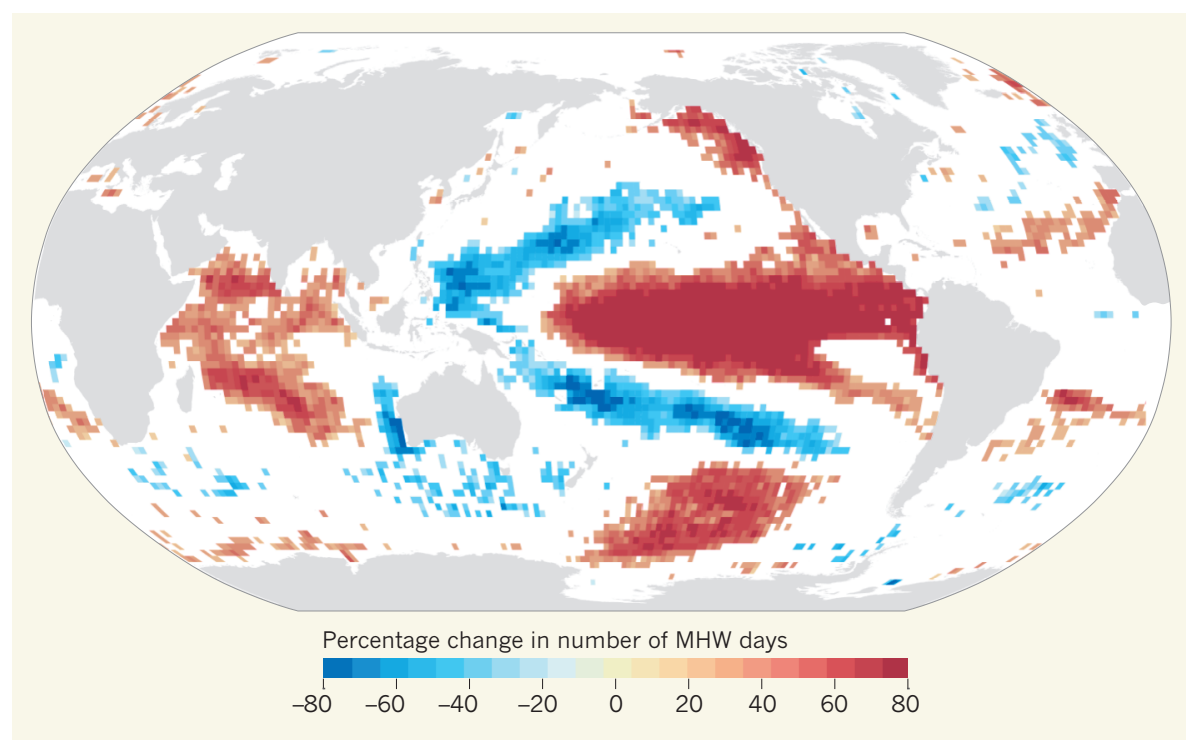

Figure 1 Climate oscillations are associated with the occurrence of marine heatwaves (MHWs). Holbrook et al. ${ }^{2}$ report a global analysis of MHWs - extended periods of anomalously warm ocean temperatures. They find that the occurrence of MHWs can be increased or suppressed during climate oscillations such as the El Niño-Southern Oscillation (ENSO), which involves episodic warming and cooling of the tropical Pacific Ocean's surface waters. This map shows the percentage increase or decrease in the number of days of MHWs across the ocean during the warm phase of ENSO, compared with the annual median percentage of MHW days, for the period from 1982 to 2016. (From Figure 3 of ref. 2.)

is used by climate-change deniers when they cite transient temperature extremes (winter storms or cold spells) as 'evidence' against the existence of long-term temperature change (global warming).

This issue of MHW definitions also muddies the relationships described by Holbrook et al., especially when extended into the future. For example, regions that experience MHWs during El Niño events similarly experience cold events during La Niña years (ENSO's cold phase). But if the shifting temperature baseline is not accounted for when defining MHWs, future cold events during La Niña years will eventually be categorized as MHWs, because even these relatively cold future periods might be warm by today's standards.

Nevertheless, there are mechanisms by which long-term climate change might alter or contribute to MHWs. Past studies have found evidence that, under climate change, there might be changes in climate oscillations such as $\mathrm{ENSO}^{8,9}$, or in the teleconnections that link these oscillations to remote areas of the ocean ${ }^{10}$. Holbrook and colleagues' work enables us to hypothesize about the global imprint of such changes on MHWs. Interestingly, patterns of change in ocean-temperature variability, and consequently in MHW intensity, might be quite different from patterns of ocean warming. Several regions historically associated with intense MHWs are projected to warm strongly until the end of the twentyfirst century, but might actually show decreased temperature variability around that warmer baseline $^{11}$. Other regions, particularly those at high latitudes, might see marked increases in temperature variability and MHW intensity, despite a weaker long-term warming trend ${ }^{11}$.

Understanding MHWs is key to assessing their impact on marine species, which react to environmental change in different ways. For example, mobile species, including many fishes, can respond to temperature changes by relocating to find favourable conditions ${ }^{12}$. These species might return after being temporarily displaced by an MHW, but long-term warming will probably shift their distributions permanently. Similarly, immobile species such as corals might recover from acute exposure to warmer temperatures (MHWs), but not from chronic exposure (long-term warming) ${ }^{13}$.

The science of MHWs, including their drivers, impacts and future evolution, is still in its infancy. Holbrook et al. provide a valuable framework for identifying causes of MHWs, one that can be built on to improve the prediction of these events and our understanding of how they will evolve with long-term climate change.

Michael G. Jacox is in the Environmental Research Division, NOAA Southwest Fisheries Science Center, Monterey, California 93940, USA. He is also at the NOAA Earth System Research Laboratory, Boulder, Colorado, and at the University of California, Santa Cruz, Santa Cruz, California.

e-mail:michael.jacox@noaa.gov

1. Smale, D. A. et al. Nature Clim. Change 9, 306-312 (2019).

2. Holbrook, N. J. et al. Nature Commun. 10, 2624 (2019).

3. Quan, X., Hoerling, M., Whitaker, J., Bates, G. \& Xu, T. J. Clim. 19, 3279-3293 (2006).

4. Jacox, M. G., Alexander, M. A., Stock, C. A. \& Hervieux, G. Clim. Dyn. https://doi.org/10.1007/ s00382-017-3608-y (2017). 
5. Oliver, E. C. et al. Nature Commun. 9, 1324 (2018).

6. Frölicher, T. L., Fischer, E. M. \& Gruber, N. Nature 560, 360-364 (2018)

7. Oliver, E. C. J. Clim. Dyn. https://doi.org/10.1007/ s00382-019-04707-2 (2019)

8. Yeh, S.-W. et al. Nature 461, 511-514 (2009)

9. Cai, W. et al. Nature Clim. Change 4,111-116 (2014).
10.Di Lorenzo, E. \& Mantua, N. Nature Clim. Change 6 1042-1047 (2016).

11.Alexander M. A et al. Elementa 6, 9 (2018).

12.Pinsky, M. L., Worm, B., Fogarty, M. J.,

Sarmiento, J. L. \& Levin, S. A. Science $\mathbf{3 4 1}$ 1239-1242 (2013).

13. Connell, J. H., Hughes, T. P. \& Wallace, C. C. Ecol. Monogr. 67, 461-488 (1997).

\title{
An early modern human outside Africa
}

\begin{abstract}
Analysis of two fossils from a Greek cave has shed light on early hominins in Eurasia. One fossil is the earliest known specimen of Homo sapiens found outside Africa; the other is a Neanderthal who lived 40,000 years later. SEE ARTICLE P.500
\end{abstract}

\section{ERIC DELSON}

$\mathrm{T}$ The origin and early dispersal of Homo sapiens has long been a subject of both popular and scholarly interest ${ }^{1}$. It is almost universally agreed that $H$. sapiens (modern humans) evolved in Africa, with the earliest known fossil representatives of our species dated to around 315,000 years ago in Morocco (at a site called Jebel Irhoud) ${ }^{2}$ and approximately 260,000 years ago in South Africa (at Florisbad) ${ }^{3}$. Stone tools comparable to those found with both of these fossils have been excavated in Kenya (at Olorgesailie) ${ }^{4}$ and dated to about 320,000 years ago. On page 500 , Harvati et al. ${ }^{5}$ describe their analysis of a fossil from Apidima Cave in southern Greece that they report to be an early modern $H$. sapiens at least 210,000 years old. This fossil is the oldest known modern human in Europe, and probably in all of Eurasia, and is more than 160,000 years older than the next oldest known European fossil of H. sapiens ${ }^{6}$.

The Apidima Cave complex was excavated in the late 1970s. Two partial crania (skulls without the lower jaw), named Apidima 1 and Apidima 2, were recovered in a single block of a type of rock called breccia. Neither fossil was previously described in detail. Apidima 2 includes the facial region of the skull and had been identified as a Neanderthal ${ }^{7}$. Apidima 1 consists of only the back of the skull and had not been previously allocated definitively to a species. Harvati and colleagues used computed tomography to scan the fossils, and generated a $3 \mathrm{D}$ virtual reconstruction of each specimen. They analysed each fossil to assess aspects of its shape, and thus to determine the fossils' similarity to those of other species.

Apidima 2 is badly damaged owing to previous breakage and distortion. Analyses of all four generated reconstructions of the fossil were consistent with it being an early Neanderthal. Apidima 1 is also damaged, but the specimen is not too badly distorted, so mirroring its right and left sides yielded a good reconstruction. The authors' extensive comparative analysis indicates that this fossil is an early member of $H$. sapiens. The posterior part of the cranium is rounded like that of $H$. sapiens, and it lacks classic Neanderthal features, such as the distinctive occipital 'chignon' - a bulge at the back of the skull that is shaped like hair tied in a bun.

Earlier dating ${ }^{8}$ of a fragment of Apidima 2 using a method called uranium-series analysis indicated a minimum age of around 160,000 years. Harvati and colleagues report a more extensive set of uranium-series dating analyses, which surprisingly reveal that Apidima 1 and Apidima 2 are of different ages, even though they were found in close proximity. Apidima 2 is around 170,000 years old well within the age range of other Neanderthal fossils found across Europe (Fig. 1). Apidima 1 is dated to be at least 210,000 years old, which is much older than any other widely accepted H. sapiens fossils found outside Africa.

This finding reveals that at least two species of hominin (humans and human relatives from the branch of the family tree after our split from chimpanzees) inhabited southeastern Europe approximately 200,000 years ago. The discovery of an $H$. sapiens fossil in Apidima raises questions about what happened to this population. Given that this $H$. sapiens existed at a time when there is substantial evidence for a Neanderthal presence at other European sites, was it part of a population that was unable to compete successfully with Neanderthals, especially in the unstable climate of that time? Perhaps one or more times, the two species replaced each other as the main hominin group present in this region.

Such patterns of replacement characterize the distribution of modern humans and Neanderthals in the Levant region of the Middle East between 250,000 and 40,000 years ago. Homo sapiens replaced Neanderthals across Europe between approximately 45,000

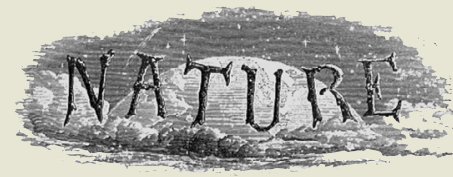

50 Years Ago

By the time the lunar samples brought back by Apollo 11 have been wrung dry of scientific information, the second American expedition to the Moon will have already been mounted ... [T] he next landing will be at one of the two sites in the eastern hemisphere which have been chosen as smooth enough for a landing ... This way by the end of the year NASA will have recovered samples of typical mare regions in both the eastern and western hemispheres of the visible face of the Moon. What the Apollo 12 astronauts ... will be instructed to look out for will depend on the first-hand descriptions of the surface radioed by Armstrong and Aldrin on Monday morning (BST) and on preliminary analyses of the samples ... Armstrong's first description that "the surface appears to be very finely grained as you get close to it, it's almost like powder" matches the Surveyor results which point to a matrix made up of finely divided particles sometimes aggregated in lumps.

From Nature 26 July 1969

100 Years Ago

The possibility of growing New Zealand flax (Phormium tenax) on a commercial scale in the British Isles has for many years been under consideration, and the publication of an important paper on the subject ... is of considerable interest... The article, which mainly consists of an account of Lord Ventry's successful experiments in co. Kerry, is illustrated by several photographs of New Zealand flax under cultivation in Ireland showing a remarkably vigorous growth ... It is pointed out in the article that only certain parts of the United Kingdom are suitable for the growth of New Zealand flax ... but as the results so far obtained are promising, it is to be hoped that every encouragement will be given to the enterprise.

From Nature 24 July 1919 